\title{
LA FORMACIÓN DE EDUCADORES BAJO UNA MODALIDAD VIRTUAL: ABORDAJE DE UN FALSO DILEMA
}

CDD: 371.39445

\section{Perla Zelmanovich}

\begin{abstract}
RESUMEN
El trabajo se estructura en cuatro apartados. El primero lleva por título "Saber poético y producción de sentidos". Valiéndose de la razón poética de María Zambrano se advierte allí sobre los riesgos de producir un vaciamiento de sentidos educativos cuando los debates se concentran en meras cuestiones técnicas. En el segundo se señalan las potencialidades que pueden brindar las nuevas tecnologías en un movimiento inverso a la segregación de sentidos en la medida que no se desentienden de las nuevas culturas generacionales post-alfabéticas. El tercer apartado aborda las presencias virtualizadas para introducir las tensiones en juego entre lo universal y lo particular que se despliegan en una iniciativa de formación en entornos virtuales. El cuarto apartado pone a disposición un breve análisis de los avatares de una experiencia singular al servicio de abordar un "falso dilema": ¿educación a distancia si o no? Allí se despliegan algunas herramientas conceptuales y metodológicas: los espacios en su estatuto de lugar simbólico; los tiempos lógicos al servicio de trabajar la posición subjetiva de los educadores en función de las apropiaciones singulares de los contenidos en juego; la relevancia del papel de Otro disponible encarnado en la figura del tutor que pueda convertir las necesidades en demandas genuinas.
\end{abstract}

\section{PALABRAS CLAVE}

Formación virtual; Saber poético; Segregación; Sentidos educativos; Posición subjetiva; Universal/Particular; Tiempos lógicos; Saber; Escritura; FLACSO

\section{TRAINING OF EDUCATORS USING A VIRTUAL MODEL: APPROACH OF A FALSE DILEMMA}

\begin{abstract}
This essay is organized into four sections. The first section is entitled "Poetic knowledge and the production of meaning". Using María Zambrano's notion of poetic reason, the section deals with the risk of producing a void in the meaning of education when debates focus merely on technical issues. In the second section, the capabilities provided by new technologies, opposed to the segregation of meaning, are pointed out, as far as they do not ignore the new generation post-alphabetic cultures. The third section deals with virtual presence in order to bring in the tension between the universal and the particular, which is displayed in an education initiative in virtual environments. The fourth section offers a brief analysis of a singular experience at the service of dealing with a "false dilemma": distance learning, yes or no? There, some conceptual and methodological tools are displayed: spaces as a symbolic place, logical times at the service of working on the subjective position of educators in accordance with the singular acquisitions of the contents at stake; the importance of the role of the Other embodied in the figure of the tutor who can turn the needs into genuine demands.
\end{abstract}

\section{KEYWORDS}

Virtual training; Poetic knowledge; Segregation; Educational senses; Subjective position, Universal/Particular; Logical times; Knowledge; Writing; FLACSO 


\section{SABER POÉTICO Y PRODUCCIÓN DE SENTIDOS}

“(...) No tener maestro es no tener a quién preguntar y, más hondamente todavía, no tener ante quién preguntarse. (...) La presencia del maestro que no ha dimitido-ni contradimitido-señala un punto, el único hacia el cual la atención dispara: El alumno se yergue. Y es ese segundo instante cuando el maestro con su quietud, ha de entregarle lo que parece imposible, ha de transmitirle, antes que un saber, un tiempo; un espacio de tiempo, un camino de tiempo. El maestro ha de llegar, como el autor, para dar tiempo y luz, los elementos esenciales de toda mediación (...) La pregunta empieza a desplegarse. La ignorancia despierta es ya inteligencia en acto. Y el maestro ha dejado de sentir el vértigo de la distancia y ese desierto de la cátedra, como todos, pródigo de tentaciones. Ignorancia y saber circulan y se despiertan igualmente por parte del maestro y del alumno, que sólo entonces empieza a ser discípulo."

Nace el diálogo.

Roma, noviembre de $1965^{1}$

Comenzar un escrito que busca preguntarse sobre la pertinencia y sobre los efectos de la educación a distancia en la formación de los educadores apelando al "saber poético"” de María Zambrano, intenta poner de relieve la cuestión de los sentidos que toda educación conlleva también cuando se trata de adultos profesionales. Postulamos que así como es necesario volver a pensar hoy los sentidos (en plural) para la educación de los niños y los jóvenes (en la pluralidad de contextos sociales, culturales y tecnológicos en las que desarrollan sus vidas) del mismo modo merecen también ser pensados los sentidos que tiene el oficio de educar y el de formarse para los adultos que tienen que sostener esa responsabilidad en los nuevos contextos que son también nuevos contextos tecnológicos. Evocamos aquí la "razón poética" de Zambrano porque tiene toda su actualidad en tanto busca hacer frente a la deshumanización de una sociedad, que para la filósofa y escritora, necesita de un saber audaz que recupere las culturas que quedan al margen y que al ser desdeñadas dejan fuera de circulación a todos aquellos que las mismas culturas producen en su condición de sujetos. Entendemos que adentrarse en las vicisitudes de las nuevas culturas tecnológicas actuales al mismo tiempo que en las desigualdades que producen y en las singularidades de los modos de relacionarse con las mismas, forma parte de ese saber audaz al

\footnotetext{
${ }^{1}$ María Zambrano (2000) "Vocación de Maestro” Editorial Ágora: Málaga. España

2 Adoptamos la expresión y la reflexión sobre el "saber poético" que aquí presentamos de la introducción que Gregorio Gómez Cambres (2000) realiza a la obra de María Zambrano en "La aurora de la razón poética".
} 
que hoy los educadores no debieran renunciar. La razón poética busca "un saber de experiencia de la vida"3 cuyo motor es un deseo de conocer que invita a que "entremos más adentro en la espesura" ${ }^{4}$, en el corazón del hombre "porque el corazón tiene razones que no conoce la razón", ${ }^{\natural,}$ según sus propias expresiones. La palabra poética es productora de pluralidad de sentidos, no sólo para quien la entrega, sino también para quien la recibe. La capacidad de producir metáforas conmueve la fijeza de la mirada única y el sinsentido (aparente) también puede ser escuchado para abrirse a significaciones insospechadas.

¿Por qué ligar las cualidades de este saber poético a las actuales transformaciones tecnológicas que aquí nos ocupan?

El sinsentido poético pone en valor un cierto vacío de saber que puede constituirse en motor de nuevas búsquedas en la medida que quien conduzca ese proceso pueda ser cada uno anclado en sus necesidades, intereses y tiempos de procesamiento singulares. En lo que atañe a la formación de los educadores, esto vale para pensar que un docente puede ser conductor de los propios procesos de formación aún en un entrono virtual, en la medida que la propuesta que se le brinde y él mismo no queden extraviados en un puro automatismo con la consecuente segregación de su propio proceso de búsqueda, de sus propios sentidos personales.

Apelar al saber poético es la manera que encontramos entonces de introducir en esta reflexión sobre los nuevos soportes tecnológicos por los que puede transitar hoy la formación de los educadores, el problema de la segregación que no es sólo material sino también simbólica, entendida aquí como segregación de sentidos. Esto requiere de abordajes que contemplen la pluralidad de particularidades que cada profesional trae consigo, es decir, requiere conmover la fijeza de la mirada sobre sus necesidades a la hora de servirnos de los beneficios de las nuevas tecnologías en tanto las mismas conmueven la relación de los sujetos con el espacio y el tiempo. Zaki Laïdi $(1997)^{6}$ nos ofrece algunas claves para pensar los

\footnotetext{
${ }^{3}$ Zambrano, M.: Hacia un saber sobre el alma. Alianza, Madrid, 1989, pág. 73.

${ }^{4}$ Zambrano, M.: "Entremos más adentro en la espesura", en Claros del bosque. Revista de Poesía y pensamiento, 1Sevilla, 1985, págs. 9-11.

5 Zambrano, M.: "La metáfora del corazón”, en Hacia un saber sobre el alma. Págs. 705-727. Alianza, Madrid, 1989, págs. 49-58.

${ }^{6}$ Zaki Laïdi es Maestro del Instituto de Estudios Políticos de París y de la Universidad John Hopkins y autor de un libro sobre el tema de la globalización, Un mundo sin sentido (FCE). Las referencias a las ideas de este autor fueron elaboradas en el Seminario II : Currículo y escuela en la trama de las instituciones. Clase Virtual: Escuela y capacitación. Variaciones acerca de los sentidos de una relación compleja. FLACSO, Argentina. Área de Educación. 2004, cuyas autoras son Silvia Finocchio y Perla Zelmanovich.
} 
sentidos de la experiencia cotidiana en el marco de una mundialización de la cultura donde la aceleración de los tiempos de las transformaciones se constituye en un aspecto crucial para entender cómo influyen los contextos alejados en el nivel local y en lo cotidiano de la vida de las personas. Señala que es posible apreciar cómo dos escalas que a primera vista parecen tan alejadas, se hallan tan íntimamente imbricadas. El tiempo mundializado uno de cuyos rasgos es la velocidad que se evidencia en la instantaneidad con que circula la información, provoca un trastrocamiento de la separación entre la experiencia y la espera.

La espera es el "entretiempo" que nos indica que puede haber una expectativa de que algo suceda, que puede haber una meta a la cual arribar. Veamos cómo el autor describe los efectos de esta separación:

(...) Como actores sociales, ya no intentamos encaminarnos a una meta, recorrer la distancia que separa la experiencia de la espera. Bajo el peso de la necesidad, pero no bajo el de la finalidad, nos vemos obligados a movernos, a circular, a comunicarnos en el espacio mundial en que ya no sólo no habría esperas, sino que, además, el campo de la experiencia se trastornaría permanentemente por la velocidad a la que nuestras prácticas, nuestros saberes o nuestros oficios se hacen obsoletos (...). (LAÏDI, 1997, p.32-33)

La ausencia de espera promociona a la necesidad como el móvil de las acciones, mientras que las finalidades se ven arrasadas por las mismas. Verificamos a diario en el cotidiano educativo así como en los espacios de formación, cómo las apremiantes necesidades le van ganando terreno a las metas, a los propósitos que le dan sentido a cualquier empresa que se precie de educativa. Si hay espera hay meta y si hay meta puede haber experiencia educativa también para los propios educadores. Cuando hay distancia que separa la experiencia de la espera la misma da sentido a los proyectos colectivos, tan difíciles de hallar en estos tiempos.

(....) como si nuestra proyección individual o colectiva en el tiempo mundial dominada por la lógica de la instantaneidad - hiciera caduca la idea misma de proyecto. Proyección, se opondría cada vez más a proyecto, como porvenir a devenir. La innovación del futuro, en cuyo nombre se había legitimado por mucho tiempo la actividad política, pierde fuerza, replegándose lastimosamente hacia la gestión del presente (... ) (LAÏDI, 1997, p.32-33)

En esta perspectiva la distancia cobra otro valor y el escenario no deseado es que los sujetos se proyecten en el porvenir, no para defender un proyecto, sino para evitar su exclusión de un juego que no comprenden cabalmente. El riesgo es que la confusión y la falta 
de diferenciación entre aquello que se hace y aquello a lo que se aspira traiga consigo la sacralización de la urgencia.

(...) Así, nuestras sociedades pretenden que la urgencia de los problemas les impide reflexionar en un proyecto, cuando en realidad es la absoluta carencia de perspectiva lo que las hace esclavas de la urgencia. La urgencia no constituye la primera etapa de un proyecto de sentido: antes bien, representa su negación activa" (...) (LAÏDI, 1997, p.32-33)

La misma lógica se traslada a los espacios de formación de los docentes y el saber poético que reintroduce aquí la cuestión del sentido nos resguarda de al menos cinco riesgos al introducir las nuevas tecnologías:

- Circunscribir el debate sobre la formación a meras cuestiones "tecnicistas".

- Desligar el "contenido" de lo que se transmite de las "formas" por las que transitan.

- Descentrar de la formación a los "sujetos" y los avatares de sus "deseos" con relación a su oficio de educar.

- Desconocer la necesidad de producir un "vínculo", de ofrecer una "interlocución" que atienda lo singular del educador que acude a la formación.

- "Burocratizar" y "estandarizar" aquello que es ofrecido.

- Someterse a la aceleración de un tiempo que no responde a los tiempos de elaboración subjetiva.

\section{INTRODUCIR LAS NUEVAS TECNOLOGÍAS EN LAS MODALIDADES DE TRANSMISIÓN, EN UN MOVIMIENTO INVERSO A LA SEGREGACIÓN DE SENTIDOS.}

Los giros culturales de los que participan de manera privilegiada las transformaciones en las tecnologías de la información y de la comunicación con sus consecuencias subjetivas, requieren repensar los modos de concebir los tiempos, los espacios y los formatos educativos a los efectos de poder responder a las nuevas lógicas preservando los sentidos, tanto para los niños y los jóvenes como para los educadores. Para los primeros, se trata de pensar espacios ricos culturalmente y dóciles a los sujetos de la "generación postalfabética”, expresión que tomamos de Franco Berardi (2007) ${ }^{7}$ para quien el concepto de generación define "un conjunto humano que comparte un ambiente de formación tecnológico y, en consecuencia, también un sistema cognitivo así como un mundo imaginario". Nos alerta

\footnotetext{
${ }^{7}$ Berardi, Franco (2008) Generación Post-alfa. Buenso Aires: Editorial Tinta Limón.
} 
con respecto a las "brechas", las distancias que esto puede suscitar en términos de transmisiones generacionales, poniendo el acento en las consecuencias que puede ocasionar hoy la aceleración de los cambios en el ambiente técnico-cultural. A diferencia de los tiempos en que pasaban décadas o quizás siglos para que las personas se familiarizasen con una técnica que modificaba sus formas de pensamiento y de acercamiento a la realidad “(...) cuando las tecnologías alfabéticas dieron paso a las tecnologías digitales, las modalidades de aprendizaje, memorización e intercambio lingüístico se modificaron rápidamente, incluso en el marco de una sola generación (...) Y los mundos generacionales comenzaron a constituirse como conjuntos cerrados, inaccesibles, incomunicables (...) por una verdadera irreductibilidad de los sistemas de referencia interpretativos".

Estas distancias generacionales nos imponen habilitar espacios que puedan abrirse a recorridos y trayectorias culturales singulares y ya no sólo apelar a la docilidad de los sujetos a las instituciones, hoy afectadas en muchos casos por una cultura que se presenta "muerta" por su pretensión conservadora y homogeneizante, en las que la falta de lugar para las experiencias nuevas y singulares atenta contra la posibilidad de establecer lazos sociales y por lo tanto vínculos educativos.

La especificidad que pensamos entonces para los espacios de formación virtual que se producen en el marco de los avatares socio-culturales y tecnológicos actuales, está regida por una orientación: producir condiciones que promuevan experiencias para todos y cada uno de los sujetos, habida cuenta de los procesos de segregación de sentidos a los que hoy asistimos al interior de las propias instituciones, segregación que también tiene el signo de una segregación "tecnológica" y "generacional". Es desde este sesgo particular donde nos ubicamos para pensar la formación en los nuevos entornos virtuales, que no pretende ser totalizadora ni excluyente de otras modalidades de formación, y que por el contrario, requiere ser matizada con formaciones presenciales.

Esta orientación que contempla la introducción de la novedad "para todos" pero que no se desentiende de los modos de procesamiento de "uno por uno", busca confrontar el trabajo educativo y la formación de los educadores con el riesgo que Michel Autès $(2004)^{8}$ propone nombrar "Riesgo de desligadura" y que atañe a la dimensión simbólica de la

\footnotetext{
${ }^{8}$ AUTÈS, Michel (2004) "Tres formas de desligadura" en Karsz, Saül (coordinador), La exclusión: bordeando sus fronteras. Definiciones y matices, Editorial Gedisa, Barcelona, pag. 31). La referencia fue tomada de la clase 10 a Cargo de Susana Brignoni, "Una experiencia de conversación entre psicoanálisis y prácticas socio-educativas: de la gestión del usuario a la producción del sujeto", del Posgrado virtual en Psicoanálisis y Prácticas Socio educativas. Aportes para abordar el Malestar educativo actual. FLACSO, sede Argentina. 2007
} 
exclusión, una de las formas contemporáneas del malestar en la cultura, leído en nuestros términos como exclusión de sentidos, del lazo social y del vínculo educativo, donde lo tecnológico juega su papel. Plantea el autor que “(...) La desligadura se realiza en lo simbólico y el resultado es una incapacidad para producir sentido y para producir sujetos (o actores)" (Autès: 31). También subraya que "La pérdida del lazo social no es pérdida de los vínculos sociales, sino del lazo identitario" (Autès: 28) y que "La exclusión es algo que afecta profundamente a la estructura identitaria de los individuos.”(Autès: 28). En esta misma dirección Autès retoma a Robert Castel cuando plantea:“(...) lo que he llamado desafiliación podría ser investigado para mostrar que no equivale necesariamente a una ausencia completa de lazos, sino también a la ausencia de inscripción del sujeto en estructuras portadoras de sentido". Los nuevos soportes tecnológicos pueden estar orientados o no, a un trabajo a favor de los procesos de ligadura simbólica, pueden constituirse o no en esos tiempos y espacios en los que se puedan construir otros vínculos culturales y sociales. Se trata de pensar las políticas de formación para que atiendan al desamparo simbólico o dicho de otro modo, para trabajar a favor del amparo cultural de los educadores, que no puede obviar su inclusión en las nuevas culturas tecnológicas de las que participan sus propios alumnos.

\section{PRESENCIAS VIRTUALIZADAS QUE TENSIONAN LA RELACIÓN ENTRE LO UNIVERSAL Y LO PARTICULAR}

Ante los riesgos de desligaduras simbólicas, los contenidos que circulan bajo el formato de clases, de apuntes, de foros, de conversaciones o de correos en las nuevas modalidades de formación virtual, no son los que harán posible por sí mismos que el saber cobre relevancia para cada uno, sino que es la presencia de un destinatario donde los sentidos de cada escrito, de cada mensaje habrán de producirse. Hete aquí el lugar estratégico que cobran los intercambios con profesores y tutores. Estas presencias virtualizadas, en las que el espacio real puede contar kilómetros de océano, nos recuerdan que la emergencia de un deseo singular que pone en función los sentidos personales de una formación, se produce a condición de que haya un reconocimiento, lo que supone una presencia de carácter simbólico. Es el reconocimiento el que introduce la dimensión de la demanda, factor crucial que es necesario considerar a la hora de activar procesos de apropiación en la formación. Para que la demanda se constituya como tal requiere ser dirigida a Otro aunque en un primer movimiento esté referida sólo a un objeto (el contenido que se brinda). Pero ese objeto en sí mismo puede 
convertirse en secundario, en la medida que la demanda es de reconocimiento, en nuestro caso, de quien se encuentra del otro lado de la pantalla.

Resguardar la relación entre saber, demanda de reconocimiento y deseo en la formación de los educadores, pretende advertir que también en la llamada educación a distancia es necesario considerar la posición subjetiva de quienes acuden a este tipo de formación. Lo que llamamos "posición" nos acerca a los modos de apropiación, que pueden expresarse bajo la forma de aceptación a-crítica, de rechazo o de apertura, entre otras. La posición subjetiva que se pone en juego es tanto de parte de quien ofrece (en nuestro caso encarnado en los profesores - tutores) como de quien recibe la transmisión (los educadores). "Ignorancia y saber circulan y se despiertan igualmente por parte del maestro y del alumno, que sólo entonces empieza a ser discípulo.”, al decir de Zambrano. (2000 [1965]). Trabajar en la formación con una modalidad virtual, es desde este punto de vista, trabajar sobre la posición subjetiva, comprometerse con los procesos de apropiación, para lo cual es necesario deja abierto un hueco, una ignorancia, un vacío necesario para hacerle lugar al deseo de cada docente que se acerca.

Una tensión se perfila hasta aquí entre un saber que requiere de su procesamiento singular y la tentación de homogeneizar la transmisión dadas las posibilidades de masividad que facilitan hoy las nuevas tecnologías. ¿Tensión entre universal (para todos) y particular (uno por uno)?

Ésta tensión, que interpela también los soportes y formatos homogéneos por los que transita hoy la educación escolar lleva a Southwell (2009) a a "realizar una exploración conceptual en torno a la tensión universalismo - particularismo". La retomamos aquí para pensar en qué medida las demandas son particulares y/o universales, y permiten democratizar la experiencia de formación. Nos recuerda Southwell que "hemos aprendido con Ernesto Laclau que el universalismo y particularismo son dos dimensiones inerradicables en la construcción de las identidades políticas, pero la forma de la articulación entre ambas está lejos de ser evidente. Plantea Laclau que "es la "globalidad" de estos proyectos (Iluministas) lo que ha entrado en crisis. Cualquiera haya de ser el signo de la nueva visión de la política que está emergiendo, está claro que una de sus dimensiones básicas habrá de ser la

\footnotetext{
9 Southwell, Myriam (2009) ¿Particular? ¿Universal?: escuela media, horizontes y comunidades. En: Revista Propuesta Educativa $\mathrm{N}^{\circ}$....FLACSO. Argentina
} 
redefinición de la relación existente entre universalidad y particularidad" (LACLAU, 1996:8)".

Hay otra tensión que se insinúa y es la que se surge de la relación entre la "distancia" que el soporte mismo construye y la "presencia" que requeriría el vínculo para dar lugar a lo singular de la demanda que la distancia estaría contrariando. "La presencia del maestro que no ha dimitido-ni contradimitido-señala un punto, el único hacia el cual la atención dispara: El alumno se yergue”. La presencia tal como la trae Zambrano - quien está abocada a pensar la vocación del maestro y que en nuestros términos abordamos como posición subjetiva - está referida a que el maestro no haya dimitido, agregamos aquí, de su deseo de educar. ¿Tensión entre distancia y presencia?

Postulamos que las tensiones entre universal y particular, entre distancia y presencia requieren ser resignificadas a partir de los avatares y las reflexiones que suscita cada experiencia de formación que se desenvuelve en un entorno virtual. Porque partimos de la premisa de que son las condiciones en las que la misma se despliega, es decir, la posición desde la cual se realiza la oferta, donde se dirime lo que entendemos se constituye en un falso dilema.

\section{AVATARES DE UNA EXPERIENCIA PARA ABORDAR UN FALSO DILEMA: ¿EDUCACIÓN A DISTANCIA SI O NO?}

La experiencia que inspira estas reflexiones recoge seis años de trabajo sostenido en la formación de educadores bajo la modalidad virtual ${ }^{10}$. Los problemas específicos de los que se parte se producen y despliegan en el marco del curso de posgrado "Psicoanálisis y prácticas socio- educativas. Aportes para Abordar el Malestar Educativo Actual" ${ }^{\text {"11. Sus }}$ destinatarios son educadores (maestros, profesores, directivos, educadores sociales) y otros profesionales que trabajan en el campo educativo (psicólogos, psicoanalistas, psicopedagogos, trabajadores sociales, abogados, funcionarios de los sistemas educativos). La propuesta tiene

\footnotetext{
${ }^{10}$ En el marco de la crisis social y económica que estalló en la Argentina los días 19 y 20 de diciembre de 2001, el Área de Educación de FLACSO inició su experiencia de brindar posgrados de formación con modalidad virtual. Quien escribe este artículo formó parte de la creación del Diploma Superior en Currículum y Prácticas Escolares en Contexto, cuya coordinación estuvo a su cargo entre los años 2005 y 2008. Desde el año 2007 dirige en la misma institución el "Programa de Investigación, formación y clínica socioeducativa" creado con un equipo de profesionales con vasta experiencia en ese campo, y del cual forma parte la propuesta virtual que se presenta en este artículo.

${ }^{11}$ El posgrado, bajo la dirección académica de Perla Zelmanovich, se ofrece en el Área de educación de FLACSO. Sede Argentina y a través de su campus virtual. El sito para acceder al mismo es: http://virtual.flacso.org.ar
} 
como horizonte dotar de toda su actualidad al malestar en la cultura educativa, articulándolo en el campo de las prácticas de cada contexto, para así dar lugar a un abordaje situado desde las preguntas particulares que cada uno se formula.

Partir de la construcción de problemas por parte de quien ofrece una formación, sea esta de carácter presencial o virtual, es un primer movimiento para trabajar sobre la posición subjetiva de sus destinatarios y supone no desentenderse de sus procesos de apropiación. Los problemas se constituyen entre una mirada sobre los obstáculos a partir del marco teórico desde el cual se los aborda y las preguntas de quienes acuden a la formación. Mientras lo que se percibe como necesidad generalmente se presenta bajo una formulación en tercera persona (“¿cómo se hace?”), para la cual se supone habría respuestas pret à porter estandarizadas, la demanda cuando se particulariza se presenta en primera persona, bajo una formulación que supone un indicio de involucramiento (“¿cómo puedo hacer yo?”). Es entre la tercera y la primera persona que algún deseo sobre la propia formación y sobre el propio oficio se puede ir conformando, a condición de que se produzca en un marco de intercambios, ya que la demanda, como vimos, para que se constituya y relance el circuito del deseo, requiere de un destinatario que la pueda escuchar.

Nos encontramos en esta experiencia de formación con lo que llamamos los "nombres del malestar", modos en que los educadores enuncian sus perplejidades y dificultades para otorgar sentidos a aquello que les acontece con los niños y los jóvenes que están llamados a educar. Hay dos enunciados que se dejan escuchar con creciente insistencia: "A mí no me prepararon para esto" y "Con estos chicos no se puede". Podemos leer en ambas formulaciones las dificultades para ligar a los sujetos en las transmisiones culturales, para producir una inscripción subjetiva.

Los espacios que se ofrecen para abordar los nombres del malestar, son entendidos en su dimensión simbólica. Las "clases" que se presentan quincenalmente son elaboradas por un cuerpo de profesores nacionales y extranjeros que participan de la producción de un campo teórico-práctico y ofrecen un "marco de ideas y conceptos actualizados y pertinentes" para una primera formulación de los problemas que se ofrecen. El "taller de escritura sobre los nombres del malestar" es una instancia central e invita a cada profesional a ingresar con una pregunta personal (el nombre del malestar) para ser trabajada a lo largo de todo el recorrido con el acompañamiento de un profesor/tutor especializado en la temática del curso. Este se constituye en ese Otro al que está destinado el mensaje. Las "conversaciones" sobre las clases 
y sobre las producciones personales del taller de escritura propician el diálogo entre colegas con la moderación del profesor/tutor. Hay una puesta en función de un lazo que gira en torno a la suposición de saber que se le atribuye al sujeto que encarna la función de tutoría. Es esta atribución la que da lugar a una regulación de la escena que se monta en ese espacio, y que la virtualidad hace transitar de manera privilegiada por la vía de la escritura. Es una instancia de compleja y delicada construcción. La sorpresa de los educadores que ingresan en esta "aventura" de transitar por diversos espacios, se produce ante la posibilidad de crear encuentros virtuales que prescindan de los cuerpos reales, facilitados por la pluralización de los recursos tecnológicos. La paradoja ante la que nos encontramos, es que lejos de saciar el deseo de producir encuentros presenciales, esta virtualización de los lazos alimenta la demanda de la presencia del Otro ${ }^{12}$. La propuesta de formación virtual atiza y potencia la creación de espacios de formación presenciales ${ }^{13}$.

Por otro lado, el lazo promocionado por la escritura en la experiencia virtual subvierte la percepción de la distancia y de la presencia. Estar allí de cuerpo presente no es necesariamente estar allí y estar allí a través de la escritura da ocasión de producir una relación de carácter simbólico, en la medida que son las ideas y las palabras las que priman para dirimir la tensión entre distancia y presencia. "C. (tutora) fue una protagonista clave del cursado y evaluación en tanto sus devoluciones eran agudas, reflexivas, tomando lo mejor del texto o de la intervención para luego arribar a lo más complejo”. (FLACSO, 2007)

Hay una lógica temporal que alimenta el proceso para que un vínculo se establezca, y que trabaja a favor de la producción de sentidos. Se ofrecen tiempos entendidos como tiempos lógicos ${ }^{14}$ para ubicar y trabajar los nombres con los que el malestar se expresa para cada uno, para que un abordaje del mismo sea posible y contempla producir sobre el final del recorrido una operación de renombrado de ese malestar. Renombrar requiere llevar a cabo una deconstrucción de los primeros enunciados, muchos de ellos firmemente sostenidos socialmente. Es un proceso que invita a que sean interrogados aquellos malestares que son de carácter general y con sentidos coagulados, que estigmatizan a los sujetos y paralizan a los profesionales, que se construyen con los significantes hegemónicos de una cultura y que se

\footnotetext{
${ }^{12}$ Nos inspiramos en el trabajo de Marcela Antelo "La sesión obsoleta", publicado en El caldero de la escuela. Disponible en www.eol.org.ar/biblioteca

${ }^{13}$ Seminarios presenciales cuya publicación está en prensa y ateneos presenciales para el trabajo con casos singulares que se abren en el ciclo de actividades 2009.

${ }^{14}$ Lacan, J. El teimpo lógico y el aserto de certidumbre anticipada. Un nuevo Sofisma. En Escritos 1. Buenos Aires: Siglo Veintiuno, Buenos Aires, 2003
} 
anudan a las trayectorias singulares de cada sujeto (aún con aparente validez científica o técnica). En los nombres, enunciados con los cuales el malestar se presenta es posible reconocer las voces de alumnos, docentes y otros profesionales que trabajan en el campo educativo, que pueden ser leídos como signos de una posición de impotencia:

\begin{abstract}
Con estos chicos no se puede, estamos muy solos, nada les interesa, nos estamos enfermando", "¿Qué hacemos con estos chicos que llegan así?", “QQué más le van a pedir a la escuela?", "Estos chicos están en otra", "¡No sabés cómo me miró!, con ellos no voy al viaje de egresados, se matan, no sé cómo separarlos", "Con estos rolingas, punk, heavy...no se puede"; "Los profes no te escuchan, no te registran";"'Lo más difícil es tratar con los padres, nos están desautorizando todo el tiempo, no se puede hacer nada si la familia no colabora, los tenés todo el tiempo en la escuela, te cuestionan todo, ésta se tornó una profesión peligrosa”;"Las autoridades no te apoyan, no contás con ellas, acá hay gente que ya tiró la toalla, que no se compromete, yo quiero hacer cosas pero acá no te dejan; cada uno está en la suya, al final cada uno termina haciendo lo que puede.
\end{abstract}

Llamamos impotencia a una posición subjetiva que asume como imposible algo que es del orden de una dificultad. Es una posición que no contribuye con la posibilidad de producir en quien la sostiene una interrogación y una implicación en el malestar que le aqueja. La apuesta de esta instancia de formación consiste en promover un recorrido que vaya del reconocimiento de una dificultad, a la ubicación de los límites propios de toda práctica, es decir, a la ubicación de aquello que resulta imposible de abordar, pero que al mismo tiempo delimita un campo de posibilidades. En este sentido, deslindar lo imposible de lo posible, es un modo de operar sobre la posición de impotencia. Se trata de comenzar a pensar en otras vías para abordar lo que aparece como detenido, yendo del obstáculo o dificultad a la producción de una posible apertura, aunque sea de carácter parcial. Cabe subrayar la diferencia entre sustraerse a las dificultades mediante el recurso a declararse impotentes y una posición que aún advertida de los límites para abordar determinadas situaciones, apuesta a una transformación posible o deseable, aunque limitada. Se trata de producir un movimiento al interior de la escena de formación, tendiente a un abordaje que involucra lo institucional (con sus límites y posibilidades), que no resuelve el todo de la cuestión en su dimensión social más amplia, lo universal, pero que tampoco queda sólo restringida a la dimensión particular de los actores involucrados. Poner a trabajar lo imposible da lugar a operar tanto sobre una posición de impotencia como de omnipotencia. La insistencia que venimos sosteniendo refiere a la necesidad de buscar algún grado de implicación personal fundada en un deseo singular en la formación. 
Este punto de partida para un itinerario que requiere de una temporalidad particular para su tratamiento, implica un primer instante de ver en toda su complejidad aquello que se presenta bajo el signo del malestar. A partir de allí se trata de dar lugar a un segundo tiempo de comprender echando mano de la teoría, que a su vez pueda llevar a construir modos de hacer en el momento de concluir, no en el sentido de cierre, sino de decisión para una acción que puede llevar una y otra vez a los dos momentos anteriores para volver a ver y a comprender. Los 2 primeros tiempos congelan el quehacer y sus preguntas en la vacilación: el mirar y la elucubración de conjeturas detienen la posibilidad del acto. El momento de concluir -que no es sin los otros momentos- supone ya la salida de una cierta vacilación. Pero este momento de concluir no entraña una construcción estratégica conciente sino que -como el sujeto- irrumpe por la vía de una certidumbre que da movimiento a la acción, pero que no es del orden de una intención absoluta, sino más bien de una apuesta que motoriza el acto, aún sin garantías. Nos orienta en este itinerario esta herramienta conceptual que adoptamos y recreamos a los efectos de tornarla productiva para nuestra tarea. El psicoanálisis plantea una distinción entre el tiempo lógico y el tiempo cronológico, unidades, que por otro lado, no coinciden. Retomando lo plantado hasta aquí, tal como lo indica el adjetivo, el tiempo "lógico" responde a una estructura dialéctica precisa que puede formularse con rigor en términos matemáticos (...) Lacan dice que el tiempo lógico tiene una estructura tripartita, cuyos tres momentos son: 1) el instante de la mirada; 2) el tiempo para comprender; 3) el momento de concluir (...) esos tres momentos no están construidos en términos de unidades cronométricas objetivas, sino como una lógica (...) basada en una tensión entre aguardar y precipitarse, entre la vacilación y la urgencia" (DYLAN, E. 2005) ${ }^{15}$. Una formación advertida con respecto a estas tensiones, cuenta con una herramienta que puede ser de gran valor para producir un intervalo, un impasse, frente a los acontecimientos que se presentan para que el precipitarse a la acción conlleve una implicación producto de ese segundo momento de elaboración.

Hay aquí una apuesta a la resignificación que va de un Sujeto impersonal (que enuncia su queja de manera difusa en el instante de ver), a un Sujeto recíproco (que lo pone a trabajar con otros en el tiempo de comprender), a un Sujeto asertivo (que reconoce de algún modo su implicación en el momento de concluir). Vemos entonces la paradoja que supone el hecho de que es en el mismo lugar en el cual el malestar se produce donde habita potencialmente su productividad. Darle entidad y especificidad a cada enunciado general y

\footnotetext{
${ }^{15}$ DYLAN, E. (2005) Diccionario Introductorio de psicoanálisis lacaniano, Buenos Aires: Paidós
} 
difuso, que se presenta como un universal ("los" chicos están en otra, "los" padres son intratables) con el cual el malestar se presenta nos lleva a poner de relieve que es necesario deconstruir ese universal, lo cual también requiere advertir el aspecto contingente del mismo, contingencia que supone detenernos en las culturas particulares, en los ideales con los que el malestar se anuda en las culturas educativas que encarnan en la singularidad de cada sujeto, de cada institución, a partir de las cuales se construyen, leen y actúan los nombres del malestar. Comprender qué está en juego en estos enunciados y en qué lógicas se sostienen, nos permitirá arribar al tercer momento para retornar de otro modo al primero, sabiendo que hay algo que es irreductible y algo sobre lo cual es posible operar, precisamente por el mismo hecho de discriminar ambos aspectos. El final del recorrido se propone pensar como un momento conclusivo, en el que se van produciendo posibles puntos de abrochamiento en los que se afirma cada educador para mirar el camino realizado y retomarlo cada vez. Establecer algunos puntos de "abrochamiento" que se pueden constituir en posibles referencias da lugar a que todo el recorrido anterior pueda resignificarse y evaluarse a partir de sus efectos. Si bien hay un trabajo y una producción realizada en un marco colectivo, el momento de concluir se juega, necesariamente, de manera individual, y en tiempos diversos y singulares para cada quien. En esto la virtualidad da una oportunidad producto de tres factores:

- La regulación individual de los tiempos (sujeto impersonal del instante de ver)

- La oportunidad de producir encuentros entre colegas de diversas latitudes en los espacios de foros (sujeto recíproco del tiempo de comprender)

- El acto conclusivo que requiere de una articulación que se ve enriquecida por el seguimiento personalizado virtual (sujeto asertivo del momento de concluir).

Los tiempos lógicos trabajan la relación de los educadores con el saber. Se agrupan a continuación tres tipos de modalidades de relación con el saber que pueden resultar orientadoras sobre los puntos de partida para el trabajo en la formación de los educadores:

- Una modalidad de aceptación a-crítica a un saber ya producido y acabado donde el sujeto y su verdad quedan excluidos. Esta modalidad se traduce en el vaciamiento de lo vivo que conlleva el saber para cada quien y la consecuente burocratización del mismo;

- Una modalidad de rechazo a la imposición de un saber que se desentiende de las verdades singulares. Esta se revela el desinterés, la apatía y el agobio del sujeto; 
- Una modalidad de apertura a nuevos saberes que pueden albergar múltiples verdades y que se traduce en la creación y recreación de la cultura en términos personales, emparentada con el saber poético.

A los efectos de trabajar sobre la posición de los profesionales con relación al saber, procuramos cuidar un rasgo fundamental de los saberes que echamos a $\operatorname{rodar}^{16}$, su carácter vivificante, es decir, su cualidad de constituirse en respuestas a preguntas abiertas, de modo tal que estén al servicio de mirar y volver a encarar la práctica cotidiana, y que permitan así encargarnos y hacernos cargo de los obstáculos. Este carácter se pone en juego a partir de la decisión de acompañar las clases con el proceso subjetivo que supone la escritura, como un modo de verificar en acto que no hay saber todo que subsuma la realidad, que no todo puede ser aprehendido, explicado, traducido. La escritura requiere elegir, dejar siempre algo afuera, aquello que se escribe no es la realidad misma. Es también un modo de dar lugar a la invención, a la recreación, y en ello va una posibilidad de implicación subjetiva. Entonces, una primera aproximación al estatuto que tiene el saber tal como lo entendemos aquí, es que es un saber que supone algún grado de implicación, y que la escritura puede jugar a favor de ese rasgo, claro que, bajo determinadas condiciones. Si tomamos lo que Lacan plantea en relación a la escritura como único modo de dar un lugar productivo al obstáculo, diremos que aquello que se escribe es lo que hace obstáculo y que, por otro lado, no hay marcas sin obstáculos. Entonces, las condiciones para una escritura en el sentido que se plantea aquí, es decir, una escritura que hace marca en quien escribe, supone algún grado de implicación. Es la escritura que pone en juego los obstáculos, que en nuestro caso atañen a nuestras prácticas socio educativas. ¿Desde qué posición se contagia el entusiasmo y se crea una atmósfera de búsqueda? "Hay que huir de lo ya sabido" dice Jacques Alain Miller. Nosotros agregamos, hay que huir de un saber cerrado, cómodo o que no nos interpela. Hasta aquí algunos rasgos que atañen al estatuto del saber que nos proponemos transmitir: un saber que tenga un carácter vivificante con respecto a las preguntas abiertas en la propia práctica; un saber que no se desentiende de los obstáculos y que interpela y apela a una implicación subjetiva para su abordaje, un saber incómodo.

A propósito de la escritura, el historiador Roger Chartier $^{17}$ (2008, p.11-13) en la lección inaugural de su cátedra consagrada al estudio de las prácticas de lo escrito en el Collège de France en octubre de 2007 plantea que “(...) las mutaciones de nuestro presente

\footnotetext{
${ }^{16}$ Kiel, Laura (2007) Clase 12. Posgrado Virtual Psicoanálisis y Prácticas Socio-educativas. FLACSO Argentina. Área de Educación.

${ }^{17}$ Chartier, Roger (2008) Escuchar a los muertos con los ojos. Katz Editores: Madrid, España.
} 
modifican todo a la vez, los soportes de la escritura, la técnica de su reproducción y diseminación, y las maneras de leer. Tal simultaneidad resulta inédita en la historia de la humanidad. La invención de la imprenta no ha modificado las estructuras elementales del libro, compuesto, tanto antes como después de Gutenberg, por pliegos, hojas y páginas reunidos en un mismo objeto (...) Al romper el antiguo lazo anudado entre los textos y objetos, entre los discursos y su materialidad, la revolución digital obliga a una radical revisión de los gestos y las nociones que asociamos a lo escrito. A pesar de la inercia del vocabulario que intenta domesticar la novedad denominándola con palabras familiares, los fragmentos de textos que aparecen en la pantalla no son páginas, sino composiciones singulares y efímeras. Y, contrariamente a sus predecesores, rollos o códices, el libro electrónico no se diferencia de las otras producciones de la escritura por la evidencia de su forma material. La discontinuidad existe incluso en las aparentes continuidades. La lectura frente a la pantalla es una lectura discontinua, segmentada, atada al fragmento más que a la totalidad. En efecto, este último invita a hojear los textos, apoyándose en los índices o bien a "saltos y brincos" como decía Montaigne. Sin embargo la similitud morfológica no debe llevar a engaño. La discontinuidad y la fragmentación de la lectura no tienen el mismo sentido cuando están acompañadas de la percepción de la totalidad textual contenida en el objeto escrito, que cuando la superficie luminosa que muestra los fragmentos de escritos no deja ver inmediatamente los límites y la coherencia del corpus de donde se lo extrajo. Los interrogantes del presente hallan sus razones en estas rupturas decisivas (...)"

Sobre el reconocimiento de estas transformaciones es que se asienta la necesidad de reubicar en estos nuevos contextos de lectura y escritura las relaciones entre las discontinuidades, la segmentación y la fragmentación que se desliga de la totalidad. Volvemos a situar aquí el papel estratégico del destinatario encarnado en el profesor-tutor, reconocido por los propios alumnos como un factor clave en este tipo de formación.

El privilegio que la propuesta de formación virtual le da a la escritura, parte a su vez del supuesto de que los "nudos" de cualquier práctica, requieren ser tratados a partir del lenguaje. Se inscribe en la perspectiva del pasaje de lo simbólico (a través de la palabra), a la ubicación de aquello que hace límite, tope, en la experiencia. Se trata de apelar y poner en función como recurso la lógica, que es la de los tres tiempos a los que ya aludimos, y es allí en donde se fundamenta el recurso a la escritura: Nombrar y narrar el malestar en el instante de ver; argumentar el malestar en tiempo de comprender; renombrar el malestar en el momento de concluir. 
A la admiración ante las increíbles promesas de navegaciones entre los archipiélagos de los textos digitales se le ha opuesto la nostalgia por un mundo de lo escrito que ya habríamos perdido. ¿Pero en verdad hay que elegir entre el entusiasmo y el lamento? (CHARTIER, 2008, p.15)

Admiración o nostalgia, entusiasmo o lamento son las palabras a las que apela Roger Chartier (2007) cuando introduce su reflexión sustentada en una perspectiva histórica, sobre las mutaciones del presente y los desafíos de la textualidad digital, que contribuye a abordar lo que planteamos aquí como falso del dilema. Del mismo modo que lo hace Berardi, reconoce la historicidad del problema y la fertilidad que tiene considerarla para su abordaje. Chartier propone "reconocer las duraciones sedimentadas de la cultura escrita para comprender más cabalmente las mutaciones que la afectan en el presente".

\section{PARA CONCLUIR}

Sostenemos que de lo que se trata hoy en una formación es buscar conmover la posición de los profesionales, de los educadores, para intentar situarlos con alguna implicación personal en su propia formación, modo en que entendemos es posible afrontar el malestar. En palabras de Maria Zambrano: "Se trata en la transmisión oral del conocimiento de un doble despertar, de una doble confluencia del saber y del no saber todavía". Paradójicamente, es en el registro de la transmisión oral que nos situamos en la formación virtual, lo cual atañe a la interpelación que se ve facilitada por la posibilidad de entablar un diálogo personal y genuino:

"Entiendo que algo del "uno por uno" se puso en juego de alguna manera en esta experiencia”. (Evaluación de educador, alumno Posgrado virtual Psicoanálisis y prácticas socio-educativas).

Coincidimos con Zambrano cuando plantea que "el alumno comienza a serlo cuando se le revela la pregunta que lleva dentro agazapada. La pregunta que es, al ser formulada, el inicio del despertar de la madurez, la expresión misma de la libertad”. 


\section{REFERENCIAS}

CHARTIER, R. Escuchar a los muertos con los ojos. Katz Editores: Madrid, 2008.

DYLAN, E. Diccionario introductorio de psicoanálisis lacaniano. Buenos Aires: Paidós, 2005

FITOUSSI, J. P.; ROSANVALLON, P. La nueva era de las desigualdades. Buenos Aires: Manantial, 1997.

GOMEZ CAMBRES, G. La aurora de la razón poética. Editorial Ágora: Málaga, 2000.

LACLAU, E. Emancipación y diferencia. Buenos Aires: Ariel, 1996.

La razón populista. Buenos Aires: Fondo de Cultura Económica, 2005.

Populismo y Transformación del Imaginario Político. Revista Todavía, Buenos Aires, n.12, dic.2005.

LAÏDI, Z. Introducción. Un mundo sin luces. In: Un mundo sin sentido. México: FCE, 1997.

SOUTHWELL, M. (2003) "El emperador está desnudo. Figuras de Modernización y Educación. In: JORNADAS "LO QUE QUEDA DE LA ESCUELA". Lo que queda de la escuela. Rosario: Laborde Editor, 2003.

. et al. Producción y reproducción de la desigualdad educativa en la escuela media: exploraciones en La Plata y zona de influencia. In: I REUNIÓN NACIONAL DE INVESTIGADORES EN JUVENTUDES. Ciudad de La Plata, 2007. Congresos y Reuniones Científicas. Artículo Completo. Ciudad de La Plata: Universidad de La Plata La Plata, 2007.

TIRAMONTI G. (Dcción.) Nuevos formatos escolares para promover la inclusión educativa. Buenos Aires: FLACSO, 2007.

Disponible en: <http://www.flacso.org.ar/educacion/investigacion_formatosesc.php $>$. Acesso em: dez.2008 
Recebido em: 16/01/2009

Publicado em: 30/06/2009 